\title{
ON PERFECT CONES AND ABSOLUTE BAIRE-ONE RETRACTS
}

\author{
Olena Karlova
}

\begin{abstract}
We introduce perfect cones over topological spaces and study their connection with absolute $B_{1}$-retracts.
\end{abstract}

\section{Introduction}

A subset $E$ of a topological space $X$ is a retract of $X$ if there exists a continuous mapping $r: X \rightarrow E$ such that $r(x)=x$ for all $x \in E$. Different modifications of this notion in which $r$ is allowed to be discontinuous (in particular, almost continuous or a Darboux function) were considered in [3], [10]-12]. The author introduced in [5] the notion of $B_{1}$-retract, i.e., a subspace $E$ of $X$ for which there exists a Baire-one mapping $r: X \rightarrow E$ with $r(x)=x$ on $E$. Moreover, the following two results were obtained in [5].

TheOREM 1.1. Let $X$ be a normal space and $E$ be an arcwise connected and locally arcwise connected metrizable $F_{\sigma}$ - and $G_{\delta}$-subspace of $X$. If

(i) E is separable, or

(ii) $X$ is collectionwise normal, then $E$ is a $B_{1}$-retract of $X$.

TheOREM 1.2. Let $X$ be a completely metrizable space and let $E$ be an arcwise connected and locally arcwise connected $G_{\delta}$-subspace of $X$. Then, $E$ is a $B_{1}$ -retract of $X$.

Note that, in the above mentioned results, $E$ is a locally arcwise connected space. Therefore, it is natural to ask

QUESTION 1.3. Is any arcwise connected $G_{\delta}$-subspace $E$ of a completely metrizable space $X$ a $B_{1}$-retract of this space?

(C) 2015 Mathematical Institute, Slovak Academy of Sciences.

2010 Mathematics Subject Classification: Primary 54C20, 54C15; Secondary 54C50. Keywords: cone over a space, $B_{1}$-retract, Baire-one mapping. 
In this work we introduce the notions of the perfect cone over a topological space and an absolute $B_{1}$-retract (see definitions in Section 2). We show that the perfect cone over a $\sigma$-compact metrizable zero-dimensional space is an absolute $B_{1}$-retract. Moreover, we give a negative answer to Question 1.3.

\section{Preliminaries}

Throughout the paper, all topological spaces have no separation axioms if it is not specified.

A mapping $f: X \rightarrow Y$ is a Baire-one mapping if there exists a sequence of continuous mappings $f_{n}: X \rightarrow Y$ which converges to $f$ pointwise on $X$.

A subset $E$ of a topological space $X$ is called:

- a $B_{1}$-retract of $X$ if there exists a sequence of continuous mappings $r_{n}: X \rightarrow E$ such that $r_{n}(x) \rightarrow r(x)$ for all $x \in X$ and $r(x)=x$ for all $x \in E$; the mapping $r: X \rightarrow E$ is called $a B_{1}$-retraction of $X$ onto $E$;

- a $\sigma$-retract of $X$ if $E=\bigcup_{n=1}^{\infty} E_{n}$, where $\left(E_{n}\right)_{n=1}^{\infty}$ is an increasing sequence of retracts of $X$;

- ambiguous if it is simultaneously $F_{\sigma}$ and $G_{\delta}$ in $X$.

A topological space $X$ is:

- perfectly normal if it is normal and every closed subset of $X$ is $G_{\delta}$;

- an absolute $B_{1}$-retract (in the class $\mathcal{C}$ of topological spaces) if $X \in \mathcal{C}$ and for any homeomorphism $h$, which maps $X$ onto a $G_{\delta}$-subset $h(X)$ of a space $Y \in \mathcal{C}$, the set $h(X)$ is a $B_{1}$-retract of $Y$; in this paper we will consider only the case $\mathcal{C}$ is the class of all perfectly normal spaces;

- a space with the regular $G_{\delta}$-diagonal if there exists a sequence $\left(G_{n}\right)_{n=1}^{\infty}$ of open neighbourhoods of the diagonal $\Delta=\{(x, x): x \in X\}$ in $X^{2}$ such that $\Delta=\bigcap_{n=1}^{\infty} G_{n}=\bigcap_{n=1}^{\infty} \bar{G}_{n}$;

- contractible if there exists a continuous mapping $\gamma: X \times[0,1] \rightarrow X$ and $x^{*} \in X$ such that $\gamma(x, 0)=x$ and $\gamma(x, 1)=x^{*}$ for all $x \in X$; the mapping $\gamma$ is called a contraction.

A family $\left(A_{s}: s \in S\right)$ of subsets of $X$ is said to be a partition of $X$ if $X=\bigcup_{s \in S} A_{s}$ and $A_{s} \cap A_{t}=\varnothing$ for all $s \neq t$.

If a space $X$ is homeomorphic to a space $Y$, then we denote this fact by $X \simeq Y$.

For a mapping $f: X \times Y \rightarrow Z$ and a point $(x, y) \in X \times Y$, let $f^{x}(y)=f_{y}(x)=$ $f(x, y)$. 


\section{ON PERFECT CONES AND ABSOLUTE BAIRE-ONE RETRACTS}

\section{Perfect cones and their properties}

The cone $\Delta(X)$ over a topological space $X$ is the quotient space $(X \times[0,1]) /$ $(X \times\{0\})$ with the quotient mapping $\lambda: X \times[0,1] \rightarrow \Delta(X)$. By $v$ we denote the vertex of the cone, i.e., $v=\lambda(X \times\{0\})$. We call the set $\lambda(X \times\{1\})$ the base of the cone.

Let $X_{1}=(0,1)$ and $X_{2}=[0,1]$. Then, $\Delta\left(X_{2}\right)$ is homeomorphic to a triangle $T \subseteq[0,1]^{2}$, while $\Delta\left(X_{1}\right)$ is not even metrizable, since there is no countable base of neighbourhoods at the cone vertex. Consequently, the naturally embedding of $\Delta\left(X_{1}\right)$ into $\Delta\left(X_{2}\right)$ is not a homeomorphism. Therefore, on the cone $\Delta(X)$ over a space $X$, one more topology $\mathcal{T}_{p}$ which coincides with the quotient topology $\mathcal{T}$ on $X \times(0,1]$ naturally appears and the base of neighbourhoods of the vertex $v$ forms the system $\{\lambda(X \times[0, \varepsilon)): \varepsilon>0\}$. The cone $\Delta(X)$ equipped with the topology $\mathcal{T}_{p}$ is said to be perfect and is denoted by $\Delta_{p}(X)$.

For all $x \in X$, we write

$$
v x=\lambda^{x}([0,1]) .
$$

Obviously,

$$
\Delta(X)=\bigcup_{x \in X} v x
$$

It is easily seen that $v x \cap v y=\{v\}$ for all distinct $x, y \in X$ and $v x \simeq[0,1]$ for all $x \in X$.

For every $y \in \Delta(X) \backslash\{v\}$, we set

$$
\alpha(y)=\operatorname{pr}_{X}\left(\lambda^{-1}(y)\right) .
$$

Obviously, $\alpha: \Delta(X) \backslash\{v\} \rightarrow X$ is continuous in both topologies $\mathcal{T}$ and $\mathcal{T}_{p}$.

Let

$$
\beta(y)= \begin{cases}\operatorname{pr}_{[0,1]}\left(\lambda^{-1}(y)\right), & y \neq v, \\ 0, & y=v .\end{cases}
$$

Then, $\beta: \Delta_{p}(X) \rightarrow[0,1]$ is a continuous function. Indeed, it is evident that $\beta$ is continuous on $\Delta_{p}(X) \backslash\{v\}$. Since

$$
\beta^{-1}([0, \varepsilon))=\lambda(X \times[0, \varepsilon))
$$

for any $\varepsilon>0$, the set $\beta^{-1}([0, \varepsilon))$ is a neighbourhood of $v$. Consequently, $\beta$ is continuous at $v$.

We observe that

$$
\lambda(\alpha(y), \beta(y))=y
$$

for all $y \in \Delta(X) \backslash\{v\}$. 


\section{OLENA KARLOVA}

\section{Remark 3.1.}

1. The concept of the perfect cone over a separable metrizable space was also defined in [8, p. 55].

2. We observe that $x \mapsto \lambda(x, 1)$ is a homeomorphism of $X$ onto $\lambda(X, \times\{1\}) \subseteq$ $\Delta_{p}(X)$. Therefore, we can identify $X$ with its image and consider $X$ as a subspace of $\Delta_{p}(X)$.

3. In the light of the previous observation, we may assume that the mapping $\alpha$ defined by formula (3.1) is a retraction.

4. The system $\left\{\beta^{-1}([0, \varepsilon)): \varepsilon>0\right\}$ is the base of neighbourhoods of the vertex of the cone according to (3.3).

Proposition $3.2([8$, p. 55]). The cone $\Delta(X)$ over a compact space $X$ is perfect.

P r o o f. Let $W$ be an open neighbourhood of $v$ in $\Delta(X)$. Then, for every $x \in X$, there exist a neighbourhood $U_{x}$ of $x$ and $\delta_{x}>0$ such that $\lambda\left(U_{x} \times\left[0, \delta_{x}\right)\right) \subseteq W$. Choose a finite subcover $\left(U_{1}, \ldots, U_{n}\right)$ of $\left(U_{x}: x \in X\right)$ and put $\varepsilon=\min \left\{\delta_{1}, \ldots, \delta_{n}\right\}$. Then, $\lambda(X \times[0, \varepsilon)) \subseteq W$. Hence, $\Delta(X)$ is the perfect cone.

Theorem 3.3. Let $X$ be a topological space.

1. If $X$ is Hausdorff, then $\Delta_{p}(X)$ is Hausdorff.

2. If $X$ is regular, then $\Delta_{p}(X)$ is regular.

3. If $X$ is a countable regular space, then $\Delta_{p}(X)$ is perfectly normal.

4. $\Delta_{p}(X)$ is contractible.

5. If $X$ is locally (arcwise) connected, then:

a) $\Delta(X)$ is locally (arcwise) connected;

b) $\Delta_{p}(X)$ is locally (arcwise) connected.

6. If $X$ is metrizable, then $\Delta_{p}(X)$ is metrizable.

Proof.

1) Let $x, y \in \Delta_{p}(X)$ and $x \neq y$. Since $\Delta_{p}(X) \backslash\{v\}$ is homeomorphic to the Hausdorff space $X \times(0,1]$, it is sufficient to consider the case $x=v$ or $y=v$. Assume that $x=v$ and $y \neq v$. Then, $0=\beta(x)<\beta(y) \leq 1$, where $\beta$ is defined by formula (3.2). Set $O_{x}=\lambda(X \times[0, \beta(y) / 2))$ and $O_{y}=\lambda(X \times(\beta(y) / 2,1])$. Then, $O_{x}$ and $O_{y}$ are disjoint neighbourhoods of $x$ and $y$ in $\Delta_{p}(X)$, respectively.

2) Fix $y \in Y$ and a closed set $F \subseteq \Delta_{p}(X)$ such that $y \notin F$. Since $X \times(0,1]$ is regular, the case $y \neq v$ and $v \notin F$ is obvious.

Let $y=v$. Choose $\varepsilon>0$ such that $F \cap \lambda(X \times[0, \varepsilon))=\varnothing$. Then, $U=$ $\lambda(X \times[0, \varepsilon / 2))$ and $V=\lambda(X \times(\varepsilon / 2,1])$ are disjoint open neighbourhoods of $v$ and $F$ in $\Delta_{p}(X)$, respectively. 


\section{ON PERFECT CONES AND ABSOLUTE BAIRE-ONE RETRACTS}

Now, let $y \neq v$ and $v \in F$. We take $\varepsilon>0$ such that $O_{v}=\beta^{-1}([0, \varepsilon))$ is an open neighbourhood of $v$ with $y \notin \bar{O}_{v}$. Moreover, since $\Delta_{p}(X) \backslash\{v\}$ is regular, there exists an open neighbourhood $O_{y}$ of $y$ in $\Delta_{p}(X)$ with $\overline{O_{y}} \cap G \subseteq \Delta_{p} \backslash F$. Then, $U=O_{y} \backslash \overline{O_{v}}$ is an open neighbourhood of $y$ in $\Delta_{p}(X)$ such that $\bar{U} \cap F=\emptyset$.

3) We observe that $\Delta_{p}(X)$ is regular by the previous proposition. Moreover, $Y$ is hereditarily Lindelöf (and, consequently, normal) as the union of countably many homeomorphic copies of $[0,1]$. Hence, $\Delta_{p}(X)$ is perfectly normal.

4) For all $y \in \Delta_{p}(X)$ and $t \in[0,1]$ define

$$
\gamma(y, t)= \begin{cases}\lambda(\alpha(y), t \cdot \beta(y)), & y \neq v, \\ v, & y=v,\end{cases}
$$

where $\alpha$ and $\beta$ are defined by (3.1) and (3.2), respectively. Then, $\gamma(y, 0)=v$ and $\gamma(y, 1)=y$ for all $y \in Y$. Clearly, $\gamma$ is continuous on $(Y \backslash\{v\}) \times[0,1]$. Let $\varepsilon>0$ and $W=\lambda(X \times[0, \varepsilon))=\beta^{-1}([0, \varepsilon))$. Since $\beta(\gamma(y, t))=t \cdot \beta(y), \gamma(W \times[0,1]) \subseteq W$. Hence, $\gamma$ is continuous at each point of the set $\{v\} \times[0,1]=\beta^{-1}(0)$.

5a) Notice that the space $\Delta(X) \backslash\{v\}$ is locally (arcwise) connected, since it is homeomorphic to the locally (arcwise) connected space $X \times(0,1]$. Let us check that $\Delta(X)$ is locally (arcwise) connected at $v$. Fix an open neighbourhood $W$ of $v$ in $\Delta(X)$. Then, $V=\lambda^{-1}(W)$ is open in $X \times[0,1]$ and $X \times\{0\} \subseteq V$. For every $x \in X$, we denote by $G_{x}$ the (arcwise) component of $V$ with $(x, 0) \in$ $G_{x}$. Then, $G_{x}$ is open in $\Delta(X) \backslash\{v\}$ and, consequently, in $\Delta(X)$. Let $G=$ $\bigcup_{x \in X} G_{x}$. Then, the set $\lambda(G)$ is an open neighbourhood of $v$ such that $\lambda(G) \subseteq W$. It remains to observe that $\lambda(G)$ is (arcwise) connected, since $v \in \lambda\left(G_{x}\right)$ and $\lambda\left(G_{x}\right)$ is (arcwise) connected for all $x \in X$.

5b) Let $\varepsilon>0$ and $W=\beta^{-1}([0, \varepsilon))$. Since each element of $W$ can be joined by a segment with $v, W$ is an arcwise connected neighbourhood of $v$.

6) Let $\varrho$ be a metric generating the topology of $X$ with $\varrho \leq 1$. For all $x, y \in X$ and $s, t \in[0,1]$ we set

$$
d(\lambda(x, s), \lambda(y, t))=|t-s|+\min \{s, t\} \varrho(x, y) .
$$

Then, $d$ is a correctly defined, symmetric, nonnegative and nondegenerate mapping of $\Delta_{p}(X) \times \Delta_{p}(X)$. Moreover, the triangle inequality is satisfied, i.e.,

$$
d(\lambda(x, s), \lambda(z, u)) \leq d(\lambda(x, s), \lambda(y, t))+d(\lambda(y, t), \lambda(z, u))
$$

for all $(x, s),(y, t),(z, u) \in X \times[0,1]$. If $t \geq \min \{s, u\}$, the inequality is obvious. Let $t<\min \{s, u\}$. Without loss of generality, we may assume that $t<u \leq s$. Then, the above inequality is equivalent to

$$
s-u+u \varrho(x, z) \leq s-t+t \varrho(x, y)+u-t+t \varrho(y, z),
$$

i.e.,

$$
\varrho(x, z) \leq 2\left(1-\frac{t}{u}\right)+\frac{t}{u}(\varrho(x, y)+\varrho(y, z)),
$$


which does hold since $\varrho(x, z) \leq 2$ and $\varrho(x, z) \leq \varrho(x, y)+\varrho(y, z)$. Moreover, $d$ generates the topology of the perfect cone. It is obvious that the $d$-neighbourhoods of $v$ are the correct ones and the metric $d$ on $\lambda(X \times(0,1])$ is equivalent to the summing metric inherited from $X \times(0,1]$.

A subset $E$ of a topological vector space $X$ is bounded if for any neighbourhood of zero $U$ there is such $\gamma>0$ that $E \subseteq \delta U$ for all $|\delta| \geq \gamma$.

Proposition 3.4. Let $Z$ be a topological vector space and $X \subseteq Z$ be a bounded set. Then $\Delta_{p}(X)$ is embedded to $Z \times \mathbb{R}$.

P r o o f. Consider the set $C=\{(x t, t): x \in X, t \in[0,1]\}$. Let $\varphi(x, t)=(x t, t)$ for all $(x, t) \in X \times[0,1]$ and $v^{*}=(0,0) \in X \times[0,1]$. Then, the restriction $\left.\varphi\right|_{X \times(0,1]}$ is a homeomorphism onto $C \backslash\left\{v^{*}\right\}$. Moreover, the mapping $\beta: C \rightarrow[0,1], \beta(z, t)=t$, is continuous. Therefore, $\beta^{-1}([0, \varepsilon))$ is an open neighbourhood of $v^{*}$ in $C$ for any $\varepsilon>0$. Now, we show that the system $\left\{\beta^{-1}([0, \varepsilon)): \varepsilon>0\right\}$ is a base of $v^{*}$. Take an open neighbourhood of zero in $Z, \delta>0$ and let $W=U \times(-\delta, \delta)$. Choose $\varepsilon \in(0, \delta)$ such that $t X \subseteq U$ for all $t$ with $|t|<\varepsilon$. Then, for each $y=(z, t) \in$ $\beta^{-1}([0, \varepsilon))$ we have $|t|<\delta$ and $z \in t x \subseteq U$. Consequently, $\beta^{-1}([0, \varepsilon)) \subseteq W$. Hence, $C$ is homeomorphic to the perfect cone $\Delta_{p}(X)$.

The following result easily follows from [8, Theorem 1.5.9].

CoRollary 3.5. The cone $\Delta_{p}(X)$ over a finite Hausdorff space $X$ is an absolute retract.

\section{Weak $B_{1}$-retracts}

A subset $E$ of a topological space $X$ is called a weak $B_{1}$-retract of $X$ if there exists a sequence of continuous mappings $r_{n}: X \rightarrow E$ such that $\lim _{n \rightarrow \infty} r_{n}(x)=x$ for all $x \in E$. Clearly, every $B_{1}$-retract is a weak $B_{1}$-retract. The converse proposition is not true (see Example 4.7).

A space $X$ is called an absolute weak $B_{1}$-retract if, for any space $Y$ and for any homeomorphic embedding $h: X \rightarrow Y$, the set $h(X)$ is a weak $B_{1}$-retract of $Y$.

Let $E=\bigcup_{n=1}^{\infty} E_{n}$ and let $\left(r_{n}\right)_{n=1}^{\infty}$ be a sequence of retractions $r_{n}: X \rightarrow E_{n}$. If the sequence $\left(E_{n}\right)_{n=1}^{\infty}$ is increasing, then $\lim _{n \rightarrow \infty} r_{n}(x)=x$ for every $x \in E$. Thus, we have proved the following fact.

Proposition 4.1. Every $\sigma$-retract of a topological space $X$ is a weak $B_{1}$-retract of $X$.

Proposition 4.2. Let $X$ be a countable Hausdorff space. Then, $\Delta_{p}(X)$ is an absolute weak $B_{1}$-retract. 


\section{ON PERFECT CONES AND ABSOLUTE BAIRE-ONE RETRACTS}

Proof. Assume that $\Delta_{p}(X)$ is a subspace of a topological space $Z$. Let $X=\left\{x_{n}: n \in \mathbb{N}\right\}$ and $X_{n}=\left\{x_{1}, \ldots, x_{n}\right\}$. Then, $\Delta_{p}(X)=\bigcup_{n=1}^{\infty} \Delta_{p}\left(X_{n}\right)$ and every $\Delta_{p}\left(X_{n}\right)$ is a retract of $Z$ by Corollary 3.5. Then, $\Delta_{p}(X)$ is a weak $B_{1}$-retract of $Z$ by Proposition 4.1

It was proved in [5] that a $B_{1}$-retract of a connected space is connected. It turns out that this is still valid for weak $B_{1}$-retracts.

TheOREm 4.3. Let $X$ be a connected space. Then, any weak $B_{1}$-retract $E$ of $X$ is connected.

P r o of. Let $\left(r_{n}\right)_{n=1}^{\infty}$ be a sequence of continuous mappings $r_{n}: X \rightarrow E$ such that $\lim _{n \rightarrow \infty} r_{n}(x)=x$ for all $x \in E$. Denote $H=\bigcup_{n=1}^{\infty} r_{n}(X)$. We show that $H$ is connected. Conversely, suppose that $H=H_{1} \cup H_{2}$, where $H_{1}$ and $H_{2}$ are disjoint sets which are closed in $H$. Observe that each set $B_{n}=r_{n}(X)$ is connected. Then, $B_{n} \subseteq H_{1}$ or $B_{n} \subseteq H_{2}$. Choose an arbitrary $x \in H_{1}$. Then, there exists a number $n_{1}$ such that $r_{n}(x) \in H_{1}$ for all $n \geq n_{1}$. Hence, $B_{n} \subseteq H_{1}$ for all $n \geq n_{1}$. Similarly, there exists a number $n_{2}$ such that $B_{n} \subseteq H_{2}$ for all $n \geq n_{2}$. Therefore, $B_{n} \subseteq H_{1} \cap H_{2}$ for all $n \geq \max \left\{n_{1}, n_{2}\right\}$, which is impossible.

It is easy to see that $H \subseteq E \subseteq \bar{H}$. Since $H$ and $\bar{H}$ are connected, $E$ is connected, too.

Lemma 4.4. Let $X$ be a normal space, $Y$ be a contractible space, $\left(F_{i}\right)_{i=1}^{n}$ be a sequence of disjoint closed subsets of $X$ and let $g_{i}: X \rightarrow Y$ be a continuous mapping for every $1 \leq i \leq n$. Then, there exists a continuous mapping $g: X \rightarrow Y$ such that $g(x)=g_{i}(x)$ on $F_{i}$ for every $1 \leq i \leq n$.

Proof. Let $y^{*} \in Y$ and $\gamma: Y \times[0,1] \rightarrow Y$ be a continuous mapping such that $\gamma(y, 0)=y$ and $\gamma(y, 1)=y^{*}$ for all $y \in Y$. For all $x, y \in Y$ and $t \in[0,1]$, define

$$
h(x, y, t)= \begin{cases}\gamma(x, 2 t), & 0 \leq t \leq 1 / 2, \\ \gamma(y,-2 t+2), & 1 / 2<t \leq 1\end{cases}
$$

Then, the mapping $h: Y \times Y \times[0,1] \rightarrow Y$ is continuous, $h(x, y, 0)=x$ and $h(x, y, 1)=y$.

Let $n=2$. By Urysohn's lemma, there is a continuous function $\varphi: X \rightarrow[0,1]$ such that $\varphi(x)=0$ on $F_{1}$ and $\varphi(x)=1$ on $F_{2}$. For all $x \in X$, let

$$
g(x)=h\left(g_{1}(x), g_{2}(x), \varphi(x)\right) .
$$

Clearly, $g: X \rightarrow Y$ is continuous and $g(x)=g_{1}(x)$ if $x \in F_{1}$, and $g(x)=g_{2}(x)$ if $x \in F_{2}$. 


\section{OLENA KARLOVA}

Assume the assertion of the lemma is true for $k$ sets, where $k=1, \ldots, n-1$, and prove it for $n$ sets. According to our assumption, there exists a continuous mapping $\tilde{g}: X \rightarrow Y$ such that $\left.\tilde{g}\right|_{F_{i}}=g_{i}$ for every $i=1, \ldots, n-1$. Since the sets $F=\bigcup_{i=1}^{n-1} F_{i}$ and $F_{n}$ are closed and disjoint, there exists a continuous mapping $g: X \rightarrow Y$ such that $\left.g\right|_{F}=\tilde{g}$ and $\left.g\right|_{F_{n}}=g_{n}$. Then, $\left.g\right|_{F_{i}}=g_{i}$ for every $1 \leq i \leq n$.

TheOREM 4.5. Let $E$ be a contractible ambiguous weak $B_{1}$-retract of a normal space $X$. Then, $E$ is a $B_{1}$-retract of $X$.

P r o of. Let $\left(r_{n}\right)_{n=1}^{\infty}$ be a sequence of continuous mappings $r_{n}: X \rightarrow E$ such that $\lim _{n \rightarrow \infty} r_{n}(x)=x$ for all $x \in E$. Choose increasing sequences $\left(E_{n}\right)_{n=1}^{\infty}$ and $\left(F_{n}\right)_{n=1}^{\infty}$ of closed subsets of $X$ such that $E=\bigcup_{n=1}^{\infty} E_{n}$ and $X \backslash E=\bigcup_{n=1}^{\infty} F_{n}$. Fix $x^{*} \in E$. Then, for every $n \in \mathbb{N}$, by Lemma 4.4 there exists a continuous mapping $f_{n}: X \rightarrow E$ such that $f_{n}(x)=r_{n}(x)$ if $x \in E_{n}$, and $f_{n}(x)=x^{*}$ if $x \in F_{n}$. It is easy to verify that the sequence $\left(f_{n}\right)_{n=1}^{\infty}$ is pointwise convergent on $X$ and $\lim _{n \rightarrow \infty} f_{n}(X) \subseteq E$. Let $r(x)=\lim _{n \rightarrow \infty} f_{n}(x)$ for all $x \in X$. Then, $r(x)=\lim _{n \rightarrow \infty} r_{n}(x)=x$ for all $x \in E$.

Proposition 4.6. The perfect cone $\Delta_{p}(X)$ over a countable regular space $X$ is an absolute $B_{1}$-retract.

P r o of. We first note that $\Delta_{p}(X)$ is perfectly normal by Theorem 3.3 (3.3). Assume that $\Delta_{p}(X)$ is a $G_{\delta^{-}}$subset of a perfectly normal space $Z$. Then, $\Delta_{p}(X)$ is a weak $B_{1}$-retract of $Z$ by Proposition 4.2 . Moreover, $\Delta_{p}(X)$ is a contractible $F_{\sigma}$-subspace of $Z$. Hence, Theorem 4.5 implies that $\Delta_{p}(X)$ is a $B_{1}$-retract of $Z$.

Let us observe that any $B_{1}$-retract of a space with a regular $G_{\delta}$-diagonal is a $G_{\delta}$-subset of this space [5, Proposition 2.2]. But it is not valid for weak $B_{1}$-retracts as the following example shows.

EXAmple 4.7. Let $\mathbb{Q}$ be a set of all rational numbers and $X=\mathbb{Q} \cap[0,1]$. Then, $\Delta_{p}(X)$ is a weak $B_{1}$-retract of $\mathbb{R}^{2}$ but not a $B_{1}$-retract of $\mathbb{R}^{2}$.

P r o of. Indeed, $\Delta_{p}(X)$ is a weak $B_{1}$-retract of $\mathbb{R}^{2}$ by Proposition 4.2 . Since $\Delta_{p}(X)$ is not a $G_{\delta^{-}}$set in $\mathbb{R}^{2}, \Delta_{p}(X)$ is not a $B_{1}$-retract.

Theorem 4.8. Let $X$ be a perfectly normal space, $E$ be a contractible $G_{\delta}$-subspace of $X, x^{*} \in E$ and let $\left(E_{n}: n \in \mathbb{N}\right)$ be a cover of $E$ such that

1. $E_{n} \cap E_{m}=\left\{x^{*}\right\}$ for all $n \neq m$;

2. $E_{n}$ is a relatively ambiguous set in $E$ for every $n$;

3. $E_{n}$ is a (weak) $B_{1}$-retract of $X$ for every $n$.

Then $E$ is a (weak) $B_{1}$-retract of $X$. 


\section{ON PERFECT CONES AND ABSOLUTE BAIRE-ONE RETRACTS}

Proof. From [6, p. 359], it follows that for every $n$ there exists an ambiguous set $C_{n}$ in $X$ such that $C_{n} \cap E=E_{n} \backslash\left\{x^{*}\right\}$. Moreover, there exists a sequence $\left(F_{n}\right)_{n=1}^{\infty}$ of closed subsets of $X$ such that $X \backslash E=\bigcup_{n=1}^{\infty} F_{n}$. Let $D_{n}=C_{n} \cup F_{n}, n \geq 1$. Now, define $X_{1}=D_{1}$ and $X_{n}=D_{n} \backslash\left(\bigcup_{k<n} D_{k}\right)$ if $n \geq 2$. Then, $\left(X_{n}: n \in \mathbb{N}\right)$ is a partition of $X \backslash\left\{x^{*}\right\}$ by ambiguous sets $X_{n}$ and $X_{n} \cap E=E_{n} \backslash\left\{x^{*}\right\}$ for every $n \geq 1$.

Suppose that $E_{n}$ is a weak $B_{1}$-retract of $X$ for every $n$. Choose a sequence $\left(r_{n, m}\right)_{m=1}^{\infty}$ of continuous mappings $r_{n, m}: X \rightarrow E_{n}$ such that $\lim _{m \rightarrow \infty} r_{n, m}(x)=x$ for all $x \in E_{n}$. Since $X_{n}$ is $F_{\sigma}$ in $X$, for every $n$, there is an increasing sequence $\left(B_{n, m}\right)_{m=1}^{\infty}$ of closed subsets $B_{n, m}$ of $X$ such that $X_{n}=\bigcup_{m=1}^{\infty} B_{n, m}$. Let $A_{n, m}=\varnothing$ if $n>m$, and $A_{n, m}=B_{n, m}$ if $n \leq m$. Then, Lemma 4.4 implies that for every $m \in \mathbb{N}$ there is a continuous mapping $r_{m}: X \rightarrow E$ such that $\left.r_{m}\right|_{A_{n, m}}=r_{n, m}$ and $r_{m}\left(x^{*}\right)=x^{*}$.

We will show that $\lim _{m \rightarrow \infty} r_{m}(x)=x$ on $E$. Fix $x \in E$. If $x=x^{*}$, then $r_{m}(x)=x$ for all $m$. If $x \neq x^{*}$, then there is a unique $n$ such that $x \in E_{n}$. Since $\left(A_{n, m}\right)_{m=1}^{\infty}$ increases, there exists a number $m_{0}$ such that $x \in A_{n, m}$ for all $m \geq m_{0}$. Hence, $\lim _{m \rightarrow \infty} r_{m}(x)=\lim _{m \rightarrow \infty} r_{n, m}(x)=x$. Therefore, $E$ is a weak $B_{1}$-retract of $X$.

If $E_{n}$ is a $B_{1}$-retract of $X$ for every $n$, we apply similar arguments.

\section{Cones over ambiguous sets}

TheOREM 5.1. Let $\Delta_{p}(X)$ be the perfect cone over a metrizable locally arcwise connected space $X, Z$ be a normal space, and let $h: \Delta_{p}(X) \rightarrow Z$ be an embedding such that $h\left(\Delta_{p}(X)\right)$ is an ambiguous set in $Z$. If

a) $X$ is separable, or

b) $\Delta_{p}(X)$ is collectionwise normal,

then $h\left(\Delta_{p}(X)\right)$ is a $B_{1}$-retract of $Z$.

Proof. We notice that $h\left(\Delta_{p}(X)\right)$ is metrizable, arcwise connected and locally arcwise connected according to Theorem 3.3. Then, the set $h\left(\Delta_{p}(X)\right)$ is a $B_{1}$-retract of $Z$ by Theorem 1.1 .

By $B_{\varepsilon}\left(x_{0}\right)$, we denote an open ball in a metric space $X$ with center at $x_{0} \in X$ and with radius $\varepsilon$.

TheOREM 5.2. Let $\Delta_{p}(X)$ be the perfect cone over a zero-dimensional metrizable separable space $X, Z$ be a normal space and let $h: \Delta_{p}(X) \rightarrow Z$ be such a homeomorphic embedding that $h\left(\Delta_{p}(X)\right)$ is a closed set in $Z$. Then $h\left(\Delta_{p}(X)\right)$ is a weak $B_{1}$-retract of $Z$. 
Proof. Without loss of generality, we may assume that $\Delta_{p}(X)$ is a closed subspace of a normal space $Z$. Consider a metric $d$ on $X$ which generates its topological structure and $(X, d)$ is a completely bounded space. For every $n \in \mathbb{N}$, there exists a finite set $A_{n} \subseteq X$ such that the family $\mathcal{B}_{n}=\left(B_{\frac{1}{n}}(a): a \in A_{n}\right)$ is a cover of $X$. Since $X$ is strongly zero-dimensional [2, Theorem 6.2.7], for every $n$ there exists a finite cover $\mathcal{U}_{n}=\left(U_{i, n}: i \in I_{n}\right)$ of $X$ by disjoint clopen sets $U_{i, n}$ which refines $\mathcal{B}_{n}$. Take an arbitrary $x_{i, n} \in U_{i, n}$ for every $n \in \mathbb{N}$ and $i \in I_{n}$. For all $x \in X$ and $n \in \mathbb{N}$, define

$$
f_{n}(x)=x_{i, n},
$$

if $x \in U_{i, n}$ for some $i \in I_{n}$. Then, every mapping $f_{n}: X \rightarrow X$ is continuous and $\lim _{n \rightarrow \infty} f_{n}(x)=x$ for all $x \in X$.

Fix $n \in \mathbb{N}$. For all $y \in \Delta_{p}(X)$, we set

$$
g_{n}(y)= \begin{cases}\lambda\left(f_{n}(\alpha(y)), \beta(y)\right) & \text { if } y \neq v, \\ v & \text { if } y=v .\end{cases}
$$

We prove that $g_{n}: \Delta_{p}(X) \rightarrow \Delta_{p}(X)$ is continuous at $y=v$. Indeed, let $\left(y_{m}\right)_{m=1}^{\infty}$ be a sequence of points $y_{m} \in Y$ such that $y_{m} \rightarrow v$. Assume that $y_{m} \neq v$ for all $m$. Show that $g_{n}\left(y_{m}\right) \rightarrow v$. Fix $\varepsilon>0$. Since $\beta\left(y_{m}\right) \rightarrow 0$, there is a number $m_{0}$ such that $\beta\left(y_{m}\right)<\varepsilon$ for all $m \geq m_{0}$. Then, $g_{n}\left(y_{m}\right)=\lambda\left(f_{n}\left(\alpha\left(y_{m}\right)\right), \beta\left(y_{m}\right)\right) \in$ $\lambda(X \times[0, \varepsilon))$ for all $m \geq m_{0}$. Hence, $g_{n}$ is continuous at $v$.

Note that $g_{n}\left(\Delta_{p}(X)\right) \subseteq K_{n}$, where $K_{n}=\bigcup_{i \in I_{n}} v x_{i, n}$. Since $K_{n}$ is a compact absolute retract by Corollary 3.5, $K_{n}$ is an absolute extensor. Taking into account that $\Delta_{p}(X)$ is closed in $Z$, we get that there exists a continuous extension $r_{n}: Z \rightarrow K_{n}$ of $g_{n}$.

It remains to show that $\lim _{n \rightarrow \infty} r_{n}(y)=y$ for all $y \in \Delta_{p}(X)$. Fix $y \in \Delta_{p}(X)$. If $y=v$, then $r_{n}(y)=g_{n}(y)=v$ for all $n \geq 1$. Let $y \neq v$. Since $\lim _{n \rightarrow \infty} f_{n}(\alpha(y))=$ $\alpha(y)$ and $\lambda$ is continuous,

$$
\lim _{n \rightarrow \infty} r_{n}(y)=\lim _{n \rightarrow \infty} \lambda\left(f_{n}(\alpha(y)), \beta(y)\right)=\lambda(\alpha(y), \beta(y))=y .
$$

Hence, $\Delta_{p}(X)$ is a weak $B_{1}$-retract of $Z$.

TheOREm 5.3. The perfect cone $\Delta_{p}(X)$ over a $\sigma$-compact zero-dimensional metrizable space $X$ is an absolute $B_{1}$-retract.

Proof. Assume that $\Delta_{p}(X)$ is a $G_{\delta}$-subspace of a perfectly normal space $Z$. Since $X$ is $\sigma$-compact, there exists an increasing sequence $\left(F_{n}\right)_{n=1}^{\infty}$ of compact subsets of $Z$ such that $X=\bigcup_{n=1}^{\infty} F_{n}$. Since for every $n \in \mathbb{N}$ the set $F_{n+1} \backslash F_{n}$ is open in the zero-dimensional metrizable separable space $F_{n+1}$, there exists a partition $\left(B_{n, m}: m \in \mathbb{N}\right)$ of $F_{n+1} \backslash F_{n}$ by relatively clopen sets $B_{n, m}$ in $F_{n+1}$. Let $\mathbb{N}^{2}=\left(n_{k}, m_{k}: k \in \mathbb{N}\right), H_{0}=F_{1}$ and let $H_{k}=B_{n_{k}, m_{k}}$ for every $k \in \mathbb{N}$. Then, the family $\left(H_{k}: k=0,1, \ldots\right)$ is a partition of $X$ by compact sets $H_{k}$. 
Fix $k \in \mathbb{N}$. Let $E_{k}=\Delta_{p}\left(H_{k}\right)$ be the perfect cone over zero-dimensional metrizable separable space $H_{k}$. Then, $E_{k}$ is a closed subset of $Z$. Therefore, $E_{k}$ is a weak $B_{1}$-retract of $Z$ by Theorem 5.2 .

Since $\Delta_{p}(X)=\bigcup_{k=1}^{\infty} E_{k}$, Theorem 4.8 implies that $\Delta_{p}(X)$ is a weak $B_{1}$-retract of $Z$. It remains to apply Theorem 4.5 .

TheOREM 5.4. The perfect cone $\Delta_{p}(X)$ over a $\sigma$-compact space $X \subseteq \mathbb{R}$ is an absolute $B_{1}$-retract.

P r o of. Suppose that $\Delta_{p}(X)$ is a $G_{\delta}$-subspace of a perfectly normal space $Z$. Since $\Delta_{p}(X)$ is $\sigma$-compact, $\Delta_{p}(X)$ is $F_{\sigma}$ in $Z$.

Let $G=\operatorname{int}_{\mathbb{R}} X, F=X \backslash G, A=\Delta_{p}(G)$ and $B=\Delta_{p}(F)$. Since $G$ and $F$ are $\sigma$-compact sets, $A$ and $B$ are $\sigma$-compact sets, too. Hence, $A$ and $B$ are ambiguous subsets of $\Delta_{p}(X)$. Consequently, $A$ and $B$ are ambiguous in $Z$. Since $G$ is metrizable locally arcwise connected separable space, $A$ is a $B_{1}$-retract of $Z$ by Theorem 5.1. Since $F$ is zero-dimensional metrizable $\sigma$-compact space, $B$ is a $B_{1}$-retract of $Z$ according to Theorem 5.3. Theorem 4.8 implies that the set $\Delta_{p}(X)=A \cup B$ is a $B_{1}$-retract of $Z$.

Note that the condition of $\sigma$-compactness of $X$ in Theorems 5.2 and 5.3 is essential (see Example 6.4).

\section{The weak local connectedness point set of $B_{1}$-retracts}

Let $(Y, d)$ be a metric space. A sequence $\left(f_{n}\right)_{n=1}^{\infty}$ of mappings $f_{n}: X \rightarrow Y$ is uniformly convergent to a mapping $f$ at a point $x_{0}$ of $X$ if for any $\varepsilon>0$ there exists a neighbourhood $U$ of $x_{0}$ and $N \in \mathbb{N}$ such that

$$
d\left(f_{n}(x), f(x)\right)<\varepsilon
$$

for all $x \in U$ and $n \geq N$. We observe that if every $f_{n}$ is continuous at $x_{0}$ and the sequence $\left(f_{n}\right)_{n=1}^{\infty}$ converges uniformly to $f$ at $x_{0}$, then $f$ is continuous at $x_{0}$.

By $R\left(\left(f_{n}\right)_{n=1}^{\infty}, f, X\right)$ we denote the set of all points of uniform convergence of the sequence $\left(f_{n}\right)_{n=1}^{\infty}$ to the mapping $f$.

The closure of a set $A$ in a subspace $E$ of a topological space $X$ is denoted by $\bar{A}^{E}$.

A space $X$ is weakly locally connected at $x_{0} \in X$ if every open neighbourhood of $x_{0}$ contains a connected (not necessarily open) neighbourhood of $x_{0}$. The set of all points of weak local connectedness of $X$ will be denoted by $W L C(X)$. 
TheOREM 6.1. Let $X$ be a locally connected space, $(E, d)$ be a metric subspace of $X$ and let $r: X \rightarrow E$ be a $B_{1}$-retraction which is a pointwise limit of a sequence of continuous mappings $r_{n}: X \rightarrow E$. Then,

$$
R\left(\left(r_{n}\right)_{n=1}^{\infty}, r, X\right) \cap E \subseteq W L C(E) .
$$

Proof. Fix $x_{0} \in R\left(\left(r_{n}\right)_{n=1}^{\infty}, r, X\right) \cap E$ and $\varepsilon>0$. Set $W=B_{\varepsilon}\left(x_{0}\right)$. Choose a neighbourhood $U_{1}$ of $x_{0}$ in $X$ and a number $n_{0}$ such that

$$
d\left(r_{n}(x), r(x)\right)<\frac{\varepsilon}{4}
$$

for all $x \in U_{1}$ and $n \geq n_{0}$. Since $r$ is continuous at $x_{0}$, there exists a neighbourhood $U_{2} \subseteq X$ of $x_{0}$ such that

$$
d\left(r(x), r\left(x_{0}\right)\right)<\frac{\varepsilon}{4}
$$

for all $x \in U_{2}$. The locally connectedness of $X$ implies that there is a connected neighbourhood $U$ of $x_{0}$ such that $U \subseteq U_{1} \cap U_{2}$. Since $\lim _{n \rightarrow \infty} r_{n}\left(x_{0}\right)=x_{0}$, there exists a number $n_{1}$ such that $r_{n}\left(x_{0}\right) \in U \cap E$ for all $n \geq n_{1}$. Let $N=\max \left\{n_{0}, n_{1}\right\}$ and

$$
F=\varlimsup_{n \geq N} r_{n}(U) \text {. }
$$

We show that $F \subseteq W$. Let $x \in U$ and $n \geq N$. Then,

$$
d\left(r_{n}(x), x_{0}\right)=d\left(r_{n}(x), r\left(x_{0}\right)\right) \leq d\left(r_{n}(x), r(x)\right)+d\left(r(x), r\left(x_{0}\right)\right)<\frac{\varepsilon}{4}+\frac{\varepsilon}{4}=\frac{\varepsilon}{2} .
$$

Thus, $r_{n}(x) \in B_{\varepsilon / 2}\left(x_{0}\right)$. Then, $\bigcup_{n \geq N} r_{n}(U) \subseteq B_{\varepsilon / 2}\left(x_{0}\right)$. Hence,

$$
F \subseteq{\overline{B_{\varepsilon / 2}\left(x_{0}\right)}}^{E} \subseteq W
$$

Moreover, $r(U) \subseteq F$, provided $\lim _{n \rightarrow \infty} r_{N+n}(x)=r(x)$ for every $x \in U$. Observe that $U \cap E=r(U \cap E) \subseteq r(U)$. Therefore,

$$
x_{0} \in U \cap E \subseteq F \subseteq W,
$$

which implies that $F$ is a closed neighbourhood of $x_{0}$ in $E$.

It remains to prove that $F$ is a connected set. To obtain a contradiction, assume that $F=F_{1} \cup F_{2}$, where $F_{1}$ and $F_{2}$ are nonempty disjoint closed subsets of $F$. Clearly, $F \cap U \neq \varnothing$.

Consider the case $F_{i} \cap U \neq \varnothing$ for $i=1,2$. The continuity of $r_{n}$ implies that $r_{n}(U)$ is a connected set for every $n \geq 1$. Since, $r_{n}(U) \subseteq F, r_{n}(U) \subseteq F_{1}$ or $r_{n}(U) \subseteq F_{2}$ for every $n \geq N$. Choose $x_{i} \in F_{i} \cap U$ for $i=1,2$. Taking into account that $\lim _{n \rightarrow \infty} r_{n}\left(x_{i}\right)=x_{i}$ for $i=1,2$, we choose a number $k \geq N$ such that $r_{n}\left(x_{i}\right) \in F_{i}$ for all $n \geq k$ and for $i=1,2$. Then, $r_{k}(U) \subseteq F_{1} \cap F_{2}$, which implies a contradiction. 
Now, let $F_{1} \cap U \neq \varnothing$ and $F_{2} \cap U=\varnothing$. Then, $U \cap E \subseteq F_{1}$. Since $r_{n}\left(x_{0}\right) \in U \cap E$, $r_{n}\left(x_{0}\right) \in F_{1}$, consequently, $r_{n}(U) \subseteq F_{1}$ for all $n \geq N$. Then, $F \subseteq \overline{F_{1}}=F_{1}$. Therefore, $F_{2}=\varnothing$, a contradiction. One can similarly prove that the case when $F_{1} \cap U=\varnothing$ and $F_{2} \cap U \neq \varnothing$ is impossible.

Hence, the set $F$ is connected and $x_{0} \in W L C(E)$.

Note that we cannot replace the set $R\left(\left(r_{n}\right)_{n=1}^{\infty}, r, X\right)$ with a wider set $C(r)$ of all points of continuity of the mapping $r$ in Theorem 6.1 as the following example shows.

EXAmple 6.2. There exists an arcwise connected closed subspace $E$ of $\mathbb{R}^{2}$ and a $B_{1}$-retraction $r: \mathbb{R}^{2} \rightarrow E$ such that $C(r) \cap E \nsubseteq W L C(E)$.

Proof. Let $a_{0}=(0 ; 0), a_{n}=\left(\frac{1}{n} ; 0\right)$ for $n \geq 1$ and $X=\left\{a_{n}: n=0,1,2, \ldots\right\}$. Denote by $v a_{n}$ the segment which connects the points $v=(1 ; 0)$ and $a_{n}$ for every $n=0,1, \ldots$ Define $E=\bigcup_{n=0}^{\infty} v a_{n}$. Then, $E$ is an arcwise connected compact subspace of $\mathbb{R}^{2}$ and $W L C(E)=\left(E \backslash v a_{0}\right) \cup\{v\}$. For all $x \in \mathbb{R}^{2}$, write

$$
r(x)= \begin{cases}x & \text { if } x \in E, \\ a_{0} & \text { if } x \notin E .\end{cases}
$$

It is easy to see that $r: \mathbb{R}^{2} \rightarrow E$ is continuous at the point $x=a_{0}$. We show that $r \in B_{1}\left(\mathbb{R}^{2}, E\right)$. Since $X \backslash E$ is $F_{\sigma}$, choose an increasing sequence of closed subsets $X_{n} \subseteq \mathbb{R}^{2}$ such that $\mathbb{R}^{2} \backslash E=\bigcup_{n=1}^{\infty} X_{n}$. Let $E_{n}=\bigcup_{k=0}^{n} v a_{k}, n \geq 1$. For every $n \in \mathbb{N}$ define $A_{n}=X_{n} \cup E_{n}$. Then, for every $n$, the set $A_{n}$ is closed in $\mathbb{R}^{2}, A_{n} \subseteq A_{n+1}$ and $\bigcup_{n=1}^{\infty} A_{n}=\mathbb{R}^{2}$. Clearly, the restriction $\left.r\right|_{A_{n}}: A_{n} \rightarrow E_{n}$ is continuous for every $n$. By the Tietze Extension Theorem there is a continuous extension $f_{n}: \mathbb{R}^{2} \rightarrow \mathbb{R}^{2}$ of $\left.r\right|_{A_{n}}$ for every $n$. Notice that for every $n$ there exists a retraction $\alpha_{n}: \mathbb{R}^{2} \rightarrow E_{n}$. Let $r_{n}=\alpha_{n} \circ f_{n}$. Then, $r_{n}: \mathbb{R}^{2} \rightarrow E_{n}$ is a continuous mapping such that $\left.r_{n}\right|_{A_{n}}=\left.r\right|_{A_{n}}$ for every $n$.

It remains to show that $\lim _{n \rightarrow \infty} r_{n}(x)=r(x)$ for all $x \in \mathbb{R}^{2}$. Indeed, fix $x \in \mathbb{R}^{2}$. Then there is a number $N$ such that $x \in A_{n}$ for all $n \geq N$. Then $r_{n}(x)=r(x)$ for all $n \geq N$. Hence, $r \in B_{1}\left(\mathbb{R}^{2}, E\right)$.

Theorem 6.3. Let $X$ be a locally connected Baire space and $E$ be a metrizable $B_{1}$-retract of $X$. Then, the set $E \backslash W L C(E)$ is of the first category in $X$.

If, moreover, $X$ has a regular $G_{\delta}$-diagonal and $E$ is dense in $X$, then $W L C(E)$ is a dense $G_{\delta}$-subset of $X$.

P r o of. Let $d$ be a metric on the set $E$ which generates its topological structure. Consider a $B_{1}$-retraction $r: X \rightarrow E$ and choose a sequence $\left(r_{n}\right)_{n=1}^{\infty}$ of continuous mappings $r_{n}: X \rightarrow E$ such that $\lim _{n \rightarrow \infty} r_{n}(x)=r(x)$ for all $x \in E$. Denote $R=R\left(\left(r_{n}\right)_{n=1}^{\infty}, r, X\right)$. Then, $R \cap E \subseteq W L C(E)$ by Theorem 6.1. According to Osgood's theorem [9], $X \backslash R$ is an $F_{\sigma}$-set of the first category in $X$. Hence, $E \backslash W L C(E)$ is a set of the first category in $X$. 


\section{OLENA KARLOVA}

Now, assume that $X$ has a regular $G_{\delta}$-diagonal and $\bar{E}=X$. It follows from [5. Proposition 2.2] that $E$ is $G_{\delta}$ in $X$. Moreover, the set $R$ is dense in $X$, since $X$ is Baire. Then, $R \cap E$ is dense in $X$. Hence, $W L C(E)$ is dense in $X$. Observe that $W L C(E)$ is a $G_{\delta}$-subset of $E$ by [7, p. 233]. Then, $W L C(E)$ is $G_{\delta}$ in $X$.

The following example gives a negative answer to Question 1.3

ExAmPLE 6.4. There exists an arcwise connected $G_{\delta}$-set $E \subseteq \mathbb{R}^{2}$ such that $E$ is the perfect cone over zero-dimensional metrizable separable space $X \subseteq \mathbb{R}$ and $E$ is not a $B_{1}$-retract of $\mathbb{R}^{2}$.

P r o o f. Let $\mathbb{I}$ be the set of irrational numbers and $X=\mathbb{I} \cap[0,1]$. Define

$$
E=\{(x t, t): x \in X, t \in[0,1]\} .
$$

Then, $E \simeq \Delta_{p}(X)$. Moreover, $E$ is an arcwise connected $G_{\delta}$-subset of $\mathbb{R}^{2}$. Clearly, $\bar{E}=[0,1]^{2}$ and $W L C(E)=\{v\}$. Therefore, Theorem 6.3 implies that $E$ is not a $B_{1}$-retract of $[0,1]^{2}$. Consequently, $E$ is not a $B_{1}$-retract of $\mathbb{R}^{2}$.

Acknowledgement. The author would like to thank the referee for his helpful and constructive comments that greatly contributed to improving the final version of the paper.

\section{REFERENCES}

[1] BORSUK, K.: Theory of Retracts. Mir, Moscow, 1971. (In Russian)

[2] EngelKing, R.: General Topology, in: Sigma Series in Pure Mathematics, Vol. 6, Revised and completed ed., Heldermann Verlag, Berlin, 1989.

[3] GARRETT, B. D.: Almost continuous retracts, in: General topology and modern analysis, Proc. Conf., Univ. California, Riverside, Calif., 1980, Academic Press, New York, 1981, pp. 229-238.

[4] JOSHI, K. D.: Introduction to General Topology, New Age International (P) Ltd., New Delhi, 2004.

[5] KARLOVA, O.: Extension of continuous functions to Baire-one functions, Real Anal. Exchange 36 (2011), 149-160.

[6] KURATOWSKI, K.: Topology, V. 1, Mir, Moscow, 1966. (In Russian)

[7] KURATOWsKi, K.: Topology, V. 2, Mir, Moscow, 1968. (In Russian)

[8] VAN MILL, J.: Infinite-Dimensional Topology. Prerequisites and Introduction. North-Holland Math. Library, Vol. 43, North-Holland, Amsterdam, 1989.

[9] OSGOOD, W. F.: Über die ungleichmässige Convergenz und die gliedweise Integration der Reihen, Nachr. Ges. Wiss. Göttingen (1896), 1986, 288-291.

[10] PAWLAK, R. J.: On some characterization of Darboux retracts, Top. Proc. 17 (1992), 197-204. 


\section{ON PERFECT CONES AND ABSOLUTE BAIRE-ONE RETRACTS}

[11] ROUSH, F.-GIBSON, R. G.-KELLUM, K. R.: Darboux retracts, Proc. Amer. Math. Soc. 79 (1980), 491-494.

[12] STALLINGS, J.: Fixed point theorems for connectivity maps, Fund. Math. 47 (1959), 249-263.

[13] URYSOHN, P.: Über die Mächtigkeit der zusammenhängenden Mengen, Math. Ann. 94 (1925), 275-293.

Received May 5, 2014

Chernivtsi National University

Department of Mathematical Analysis

Kotsjubyns'koho 2

Chernivtsi 58012

UKRAINE

E-mail: maslenizza.ua@gmail.com 